\title{
Development and Modeling of Miniaturized Traveling-Wave Tubes in Millimeter and Sub-THz Bands
}

\author{
Nikita M. Ryskin ${ }^{1,2}$ \\ ${ }^{1}$ Saratov Branch, V.A. Kotel'nikov Institute of Radio Engineering and Electronics RAS, Saratov, Russia \\ ${ }^{1}$ Saratov State University, Saratov, Russia, RyskinNM@info.sgu.ru
}

\section{Introduction}

Development of compact vacuum-tube millimeter- and THz-band sources with 10-100 W output power would have a great potential for numerous applications such as high-data-rate wireless communications, security, spectroscopy, biomedical applications, etc. Among the vacuum tubes, microfabricated analogs of traveling-wave tube (TWT) amplifiers are the most promising. Over 50-W output power in G-band $(0.22 \mathrm{THz})$ has recently been demonstrated [1,2].

Slow-wave structure (SWS) is a core part of a TWT serving to slow the electromagnetic wave down to the electron beam velocity. In this paper, we present the results of recent studies aimed at modeling and development of medium power TWTs with various SWS suitable for operation in millimeter and sub$\mathrm{THz}$ frequency bands. Different types of TWTs are considered, such as folded-waveguide TWT, TWTs with sheet electron beam and dual-grating SWS, and TWTs with planar meander-line SWS on a dielectric substrate.

\section{Folded-waveguide TWT}

TWT with folded-waveguide (FW) SWS has numerous advantages due to its high coupling impedance, reasonably wide $(\sim 30 \%)$ bandwidth, relatively simple structure compatible with existing microfabrication technologies, thermal and mechanical robustness, and simple input/output coupling.

We designed and simulated FW TWTs operating at Q-band (38-54 GHz), V-band (48-90 GHz), and Gband $(175-275 \mathrm{GHz})$. Electromagnetic parameters of the SWSs were calculated by using ANSYS HFSS simlator. FW SWS circuits at Q- and V-band were fabricated using CNC-milling machine. In Fig. 1, a picture of the 50-pitch Q-band SWS with input/output couplers is shown. Cold-test electromagnetic measurements of $S$-parameters of the circuits were performed using Keysight Technologies PNA N5227A vector network analyzer. Good transmission characteristics were measured. In particular, return loss (S11) is less than $-10 \mathrm{~dB}$ and transmission loss (S21) is about $-2 \mathrm{~dB}$ in the most part of the passband. Experimental measurements are in good agreement with numerical simulations using ANSYS HFSS.

In addition, small-signal and large-signal gain regimes of Q- and V-band FW TWT amplifiers driven by a $50-\mathrm{mA}, 15-\mathrm{kV}$ electron beam were simulated using the 1-D parametric code [3]. Parameters of the tubes were obtained, for which 30-40 dB small-signal gain and nearly $100 \mathrm{~W}$ saturated power are attained.

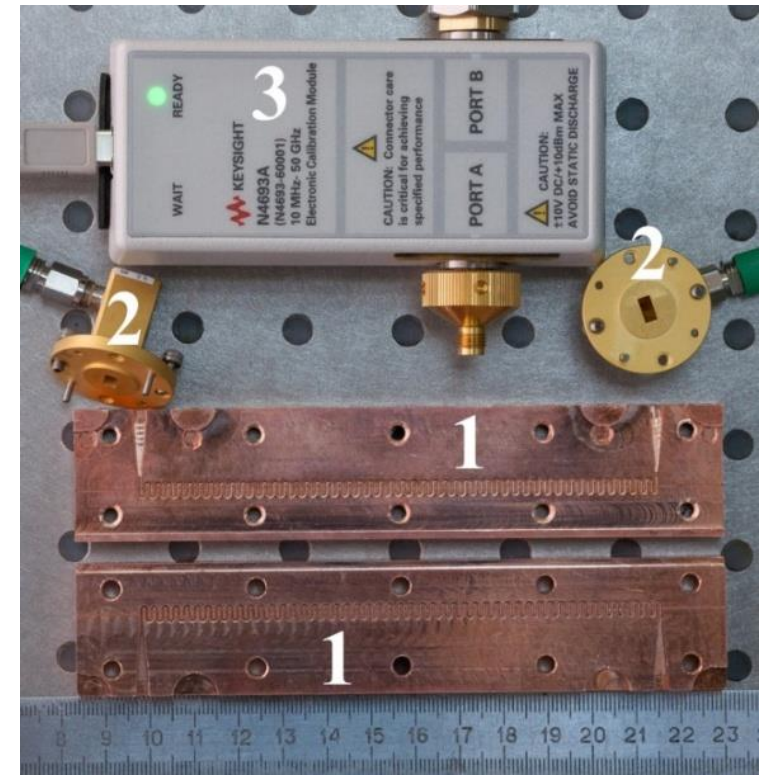

Fig. 1. Photo of the Q-band folded-waveguide SWS sample. 1 - folded waveguide; 2 - coax to waveguide adapters; 3 electronic calibration module (ECal module)

\section{Sheet-beam TWT dual-grating SWS}

Using high-aspect-ratio sheet electron beams allows substantial reduce of current density and facilitates beam focusing and transportation in a narrow beam tunnel. In $[3,4]$, we studied feasibility of a $0.2-\mathrm{THz}$ sheet-beam TWT amplifier with a staggered grating SWS. An electron gun with a $0.7 \times 0.1 \mathrm{~mm}^{2}$ thermionic cathode producing intensive sheet electron beam with over $120 \mathrm{~A} / \mathrm{cm}^{2}$ current density has been developed [3,4]. However, such a gun can operate only in a short-pulse mode with 10 us pulse duration and 2000 off-duty cycle. At the same time, the required focusing magnetic field is as high as $1.12 \mathrm{~T}$.

In order to overcome the problems mentioned above, we designed the electron-optic system (EOS) with a converging sheet electron beam having 10times compression in vertical direction using the method of synthesis described in [5]. This allows a significant reduce of the beam thickness as well as of the cathode load and focusing magnetic field $(0.8 \mathrm{~T})$. Schematic of the electron gun is presented in Fig. 2. The design was verified by 3-D simulation using LORENTZ-3EM simulator [6]. The results are in a fairly good agreement, except that the beam thickness obtained in 3-D analysis is somewhat higher than expected ( $\sim 75 \mathrm{um})$ that can be attributed to thermal and angle spread of the emitted electrons.

The gun was fabricated and up to $140-\mathrm{mA}$ beam current was measured. 


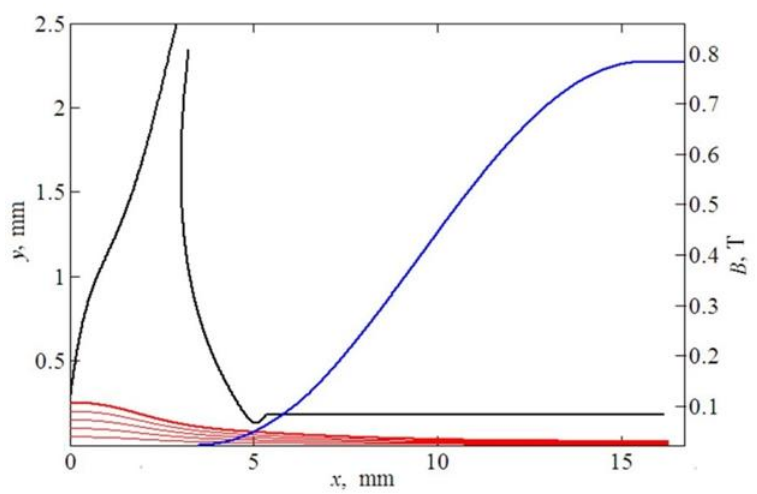

Fig. 2. Configuration of the gun electrodes, particle trajectories, and the magnetic field profile

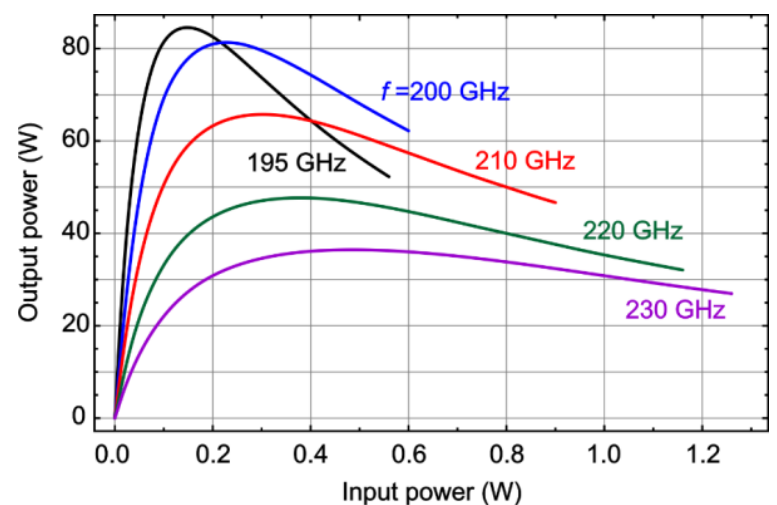

Fig. 3. Output power versus input power of the sheet-beam TWT at different frequencies

Performance of the TWT with 0.1-A, 20-kV sheet beam and dual-grating SWS was simulated. Parameters of the SWS were the same as in $[3,4]$ except the beam tunnel height, which was reduced from 200 um to $150 \mathrm{um}$, owing to reduced beam thickness. This results in significant increase of the coupling impedance and the gain factor. For the 40-mm-length TWT circuit, over 30-dB small-signal gain was observed in the simulations (instead of $\sim 20 \mathrm{~dB}$ in [3]). In Fig. 3, output power versus input power at different frequencies is plotted. Saturated power over $80 \mathrm{~W}$ is observed, which is close to [3]. However, the saturation is attained at the input power about $0.1 \mathrm{~W}$, which is nearly 10 times less than in [3].

\section{TWT with planar microstrip meander-line SWS}

In order to reduce the tube size and dimensions, as well as the dc voltage, planar microstrip meanderline SWS on dielectric substrates have been proposed. We fabricated SWS circuits in V-and W-band using the novel technology utilizing magnetron sputtering and laser ablation methods [7]. Several samples of Vband and W-band meander SWS circuits on quartz substrates were fabricated. The strip thickness was increased up to $10 \mathrm{um}$ that should increase robustness of the circuits. In Fig. 4, photo of the 50-pitch SWSs with matching elements at both ends is shown.

Cold-test measurement of the electromagnetic parameters shows good transmission characteristics. Measured transmission loss of the structure did not exceed $-2.5 \mathrm{~dB}$.

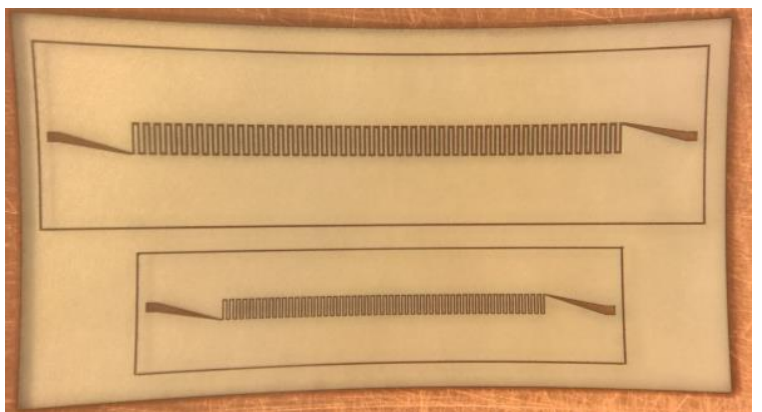

Fig. 4. Photo of the V-and W-band meander SWS samples fabricated by laser ablation

The developed SWSs provide high slow-down factor $\left(c / v_{p h} \sim 7 \div 9\right)$ and thus are suitable for operation with a low-voltage $(3 \div 5 \mathrm{kV})$ electron beam. Small-signal and large-signal gain characteristics of the TWT driven by a 100-mA sheet electron beam were simulated. For a 1-cm-length SWS, the peak gain is about $15-20 \mathrm{~dB}$. Owing to strong dispersion of the meander-line SWS, the bandwidth of the amplifier is rather narrow $(2-3 \mathrm{GHz})$. However, the central frequency may be easily tuned in a wide range by varying the beam voltage. The simulations predict over 60 $\mathrm{W}$ output power at saturation.

\section{Acknowledgment}

This research is supported by Russian Science Foundation under Grant \# 17-12-01160.

\section{References}

1. Joye C.D., Cook A.M., Calame J.P., Abe D.K.,et al. Demonstration of a high power, wideband $220-\mathrm{GHz}$ traveling wave amplifier fabricated by UV-LIGA // IEEE Trans. Electron Devices. 2014. V. 61, No. 6, P. 1672-1678.

2. Baig A., Gamzina D., Kimura T., Atkinson J. et al., Performance of a nano-CNC machined $220-\mathrm{GHz}$ traveling wave tube amplifier // IEEE Trans. Electron Devices. 2017. V. 64, No. 6. P. 2390-2397.

3. Karetnikova T.A., Rozhnev A.G., Ryskin N.M., Fedotov A.E., Mishakin S.V., Ginzburg N.S. Gain analysis of a 0.2-THz traveling-wave tube with sheet electron beam and staggered grating slow wave structure / // IEEE Trans. Electron Devices. 2018. V. 65, No. 6. P. 2129-2134.

4. Karetnikova T.A., Rozhnev A.G., Ryskin N.M., Torgashov G.V. et al. Modeling a subterahertz travelingwave tube with a slow-wave structure of the double grating type and a sheet electron beam // J. Commun. Technol. Electron. 2016. V. 61, No. 1. P. 50-55.

5. Yu.G. Gamayunov, E.V. Patrusheva. Synthesis of systems formation of converging sheet electron beams at partial magnetic shielding of the cathode // J. Commun. Technol. Electronics. 2017. Vol. 62, No. 11, 1291-1297.

6. Karetnikova T.A., Rozhnev A.G., Ryskin N.M., Burtsev A.A., Navrotsky I.A., Danilushkin A.V. Design and simulation of a $0.2-\mathrm{THz}$ traveling-wave tube with a converging sheet electron beam // Proc. 19th International Vacuum Electronics Conference (IVEC 2018). 24-26 Apr. 2018, Monterey, CA, USA. P. 161-162.

7. Ryskin N.M., Rozhnev A.G., Starodubov A.V., Serdobintsev A.A., et al. Planar microstrip slow-wave structure for a low-voltage V-band traveling-wave tube with a sheet electron beam // IEEE Electron Device Lett. 2018. V. 39, No. 5. P. 757-760. 